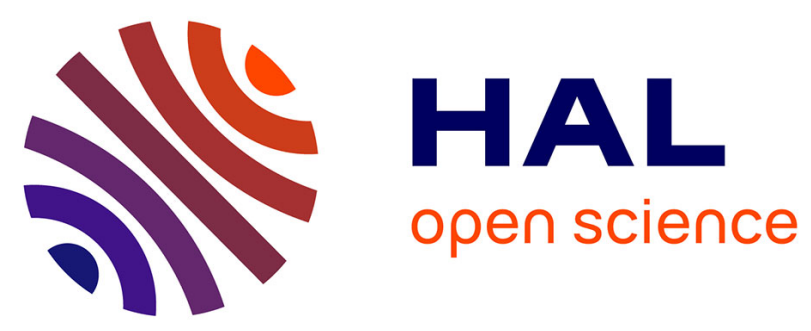

\title{
Dependable multi-touch interactions in safety critical industrial contexts: Application to aeronautics
}

\author{
Arnaud Hamon, Philippe Palanque, Martin Cronel
}

\section{To cite this version:}

Arnaud Hamon, Philippe Palanque, Martin Cronel. Dependable multi-touch interactions in safety critical industrial contexts: Application to aeronautics. IEEE 13th International Conference on Industrial Informatics (INDIN 2015), IEEE, Jul 2015, Cambridge, United Kingdom. pp.980-987, 10.1109/INDIN.2015.7281868 . hal-03209328

\section{HAL Id: hal-03209328 https://hal.science/hal-03209328}

Submitted on 29 Apr 2021

HAL is a multi-disciplinary open access archive for the deposit and dissemination of scientific research documents, whether they are published or not. The documents may come from teaching and research institutions in France or abroad, or from public or private research centers.
L'archive ouverte pluridisciplinaire HAL, est destinée au dépôt et à la diffusion de documents scientifiques de niveau recherche, publiés ou non, émanant des établissements d'enseignement et de recherche français ou étrangers, des laboratoires publics ou privés. 


\title{
Dependable Multi-Touch Interactions in Safety Critical Industrial Contexts: Application to Aeronautics
}

\author{
Arnaud Hamon, Philippe Palanque \& Martin Cronel \\ Interactive Critical Systems Group, Université de Toulouse \\ Toulouse, France \\ hamon@irit.fr, palanque@irit.fr,cronel@irit.fr
}

\begin{abstract}
Over the last few years multi-touch interfaces have made their ways in most environments including mobile technologies, flight entertainment systems, consumer electronics ... Such interfaces and associated interaction techniques have demonstrated benefits partly due to the fact that the output device integrates input management thus bridging the (classical) gap between input and output in user interfaces. They have also demonstrated benefits in terms of performance for triggering commands by exploiting multi-fingers interactions thus reducing the number of unnecessary modes. Together with these benefits, multi-touch interfaces bring a set of issues that are still to be solved prior to make them "acceptable" for command and control of (safety) critical interactive systems. Such issues include usability and reliability in a way that is even more salient than in "classical" Windows, Icons, Menus, Pointing device (WIMP) interfaces that have been around for more than 30 years. This paper proposes a notation and its associated tool for describing in a complete and unambiguous way multi-touch interactions thus mainly targeting at reliability. We present from a case study (in the application domain of interactive cockpits of aircrafts) how the notation makes it possible to describe such interactions from the hardware level and how this notation can be integrated into an industrial development process.
\end{abstract}

Keywords—multi-touch interaction, cockpits, aeronautics

\section{INTRODUCTION}

Over the years, the evolution of cockpits in large civil aircrafts has taken place following two different paths:

- Small increments/evolutions targeting at solving identified problems or integrating new equipment into an existing cockpit,

- Significant steps/evolutions ending up with a complete redesign of the cockpits including controls and displays. Examples of such evolutions include glass cockpit (where large display units were included in the flight deck) and more recently interactive cockpits compliant with Aeronautical Radio, Incorporated (ARINC) 661 specifications [3] where interaction takes place through mouse-like input devices and keyboards.

In parallel of these evolutions in the cockpit, multi-touch interfaces have made their ways in most environments including mobile technologies, flight entertainment systems, consumer electronics ... Such interfaces have demonstrated benefits related to the fact that the output device integrates input management thus bridging the (usual) gap between input and output in user interfaces. They have also demonstrated benefits in terms of performance for triggering commands by exploiting multi-fingers interactions thus reducing the number of un-necessary modes.

Providing multi-touch interactions for the command and control of civil aircraft would consists in another significant evolutionary step as far as the evolution of cockpits is concerned. However, together with the benefits presented above, multi-touch interfaces bring a set of issues that are still to be solved prior making them "acceptable" for command and control of (safety) critical interactive systems. These issues touch many properties such as reliability, and more globally resilience.

These sets of issues are not new and appeared already when interactions in the cockpit evolved from "physical interactions" (by manipulating physical knobs and reading information on dials) to software controls mainly based on the ARINC 661 specification [3]. Often considered more reliable means to trigger commands from the cockpit equipment's, pushbuttons, rotators and safe-guarded physical buttons have populated the flight decks' space. However these devices generate significant weight load and bring modifiability (upgradability) issues. Indeed, these physical components are directly linked to the systems they command resulting in the fact that evolutions made to the aforementioned systems are likely to require modifications to the components. This requires adaptation of the cockpit itself (which is a distributed cognitive system [20]) and ultimately the adaptation of procedures and training. Finally, due to their physical nature, the more complex the aircraft systems, the more numerous physical components will appear in the cockpits. The development of the ARINC 661 specification [3], described in the related work paragraph, was mainly driven by these issues i.e. reducing cockpit size and increasing its modifiability.

The remainder of this paper is organized as follows: first it addresses the rationale for the use of multi-touch applications in avionic applications as well as the related issues from a specification perspective. It then presents why multi-touch interactions have the potential for going beyond the numerous 
benefits brought by interactive cockpits compliant with ARINC 661 specification but also the new problems they raise in terms of engineering. The paper then proposes a contribution targeting at these issues and finally demonstrates on a case study how the approach can be applied, integrated in an industrial development process and how it solves the identified issues.

\section{RATIONALE FOR INVESTIGATING MULTI-TOUCH INTERACTIONS IN COCKPITS}

The evolutions brought by user interfaces compliant with ARINC 661 specification [3] have not been able to solve all the issues raised by interactions in the cockpit:

- $\quad$ Despite the potential use of Display Units (DUs i.e. computer screens) combined with Keyboard and Cursor Control Units (KCCUs), the cockpit space remains confined and a bottleneck for the ever growing complexity of aircrafts;

- Input and output are still disjoints (e.g. KCCUs and DUs) which introduces articulatory distance for pilots to control the avionic systems;

- Even though some cockpits offer touch interactions they remain limited to mono-touch techniques over WIMP interfaces.

Since the observations made in [18] several studies and research project have studied the potential benefits of multitouch interactions in cockpits. For instance, the Federal Aviation Administration (FAA) has appointed Honeywell to study the human factors aspects of pilots' activity supported by touchscreens [31]. From a manufacturer point, the Airbus R\&T cockpit division [4] studied the introduction of multi-touch displays in cockpits, following a human centred approach where a classification of multi-touch tasks according to their ergonomic criteria is proposed. That study demonstrates the benefits of using multi-touch interfaces in cockpits and the large impact the context of use has on the interaction.

Multi-user interactions extend the interaction space compared to mono-user ones. Having two users implies, for instance, that there are now four hands which can interact with the system at the same time and/or location. Following this policy it is possible to design specific interactions for a critical operation which would require actions to be performed by two operators simultaneously. For instance, a double check action with four hands in a delimited range of time [10] where both members of the crew have to touch the screen with their thumbs. The interaction techniques could also be different for the two users, e.g. the captain has to touch with both thumbs while the first officer has to make two circles at the same moment to avoid spurious and human error.

\section{ENGINEERING ISSUES FOR MULTI-TOUCH INTERACTIONS}

This section introduces the main issues for adding multi-touch interactions in cockpits from an engineering point of view. Since multi-touch interactions are multi-modal, next paragraph addresses the generic issues for engineering multimodal interactions while the second one details specific multi- touch issues. The second paragraph addresses the issues related to engineering such interaction techniques in the specific context of interactive cockpits.

\section{A. Multi-modal interactions}

As the engineering of multimodal interfaces has been studied for many years, we focus here on issues related to having more than one user involved in the interaction. We have identified two main issues related to multi-users interactions. First, for the same input device and the same input action, two users can have different interaction techniques e.g. swipe versus simple press on a touch-screen.

Second, the devices used by the various users may be of different types, not co-located and installed on different partitions running on an IMA (Integrated Modular Avionics) with different window managements. All these issues require the careful definition of multi-modal multi-user interactions making explicit the user, the input device and the interaction technique used.

Currently, our work focuses on identical interaction techniques per type of device, each person of the crew having his/her own touch-screen and a shared one (as this proposes a good combination of the issues above).

\section{B. Multi-touch interactions}

Multi-touch interaction techniques are, by definition, multimodals and therefore inherit the issues we presented in the previous paragraphs. However, they present additional specific issues and the following paragraph describes them with an emphasis on multi-touch interaction specification: interactivity continuity, dynamic instantiation, complex time relationships and finger clustering. Most of them were mentioned in [19] but interaction continuity and anthropomorphic characteristics have been recently identified.

1) Interaction continuity (issue 1)

One major evolution from the use of ARINC 661 WIMP interactions consists in describing continuous interactions (as identified in direct manipulation [36] or post-WIMP interactions [40]) the users' interactions with the system are no longer discrete (one event triggers one command) and become continuous (a flow of event is produced before triggering a command) and continuous feedback has to be produced. Consequently, the notations to describe these techniques shall support the description of event streams as well as the description of the related immediate feedbacks. Commands such as pinch to zoom belong to this category.

2) Dynamic instantiation of input devices (issue 2)

When all input devices are not known when the user interface is initialized, the input devices detected during the interaction need to be dynamically instantiated in order to be registered and listened to (i.e. their events are possibly used for triggering commands). This is typically the case while addressing multi-touch interaction techniques since users' fingers are only detected as they touch (or approach) the tactile surfaces. This is very different from WIMP interfaces where the set of mice and keyboard is defined in a static way.

3) Temporal representations and fine tuning (issues 3, 4) 
As illustrated in [19], specifying multi-touch interaction techniques require both qualitative and quantitative time descriptions. Such timing aspects (which have to be finely tuned) are required to be explicitly and thoroughly defined for describing multi-touch and multi-modal interactions as presented in [35]. For example, to specify a double tap, designers need a fine tuning on two non-consecutive events: first finger's touch and last finger's release.

4) Finger clustering and anthropomorphic characteristics (issues 5, 6)

In order to fully incorporate the expressive power of multitouch interactions there is a need to be able to detect (at interaction time) group of fingers evolving jointly. This might be the case for fingers belonging to the same hand (often called finger clustering) but this usually require specific algorithms as proposed in [8] that must be incorporable in the user interface description language. More fine grain interactions might need to analyse the fingertip shapes (to determine orientation of the finger on the touch device as proposed in [11]. Lastly, it is important to be able to describe interaction techniques taking into account the anthropomorphic characteristics of the hand [29]. For instance, an interaction featuring two fingers from the same hand will not have the same spatial and behavioural properties as an interaction with fingers belonging different hands.

\section{Multi-touch interactions in cockpits}

The avionic software environment in cockpits of civil aircrafts requires applying dedicated design processes to ensure the reliability, safety and usability of these critical embedded applications. Therefore, specifying multi-touch interaction techniques for cockpits in a complete and unambiguous way is a mandatory requirement.

1) Interaction accuracy versus usability (issue 7)

Due to the instability of the environment, it is important to be able to avoid spurious interactions (i.e. a pilot triggering inadvertently a command through a non-desired touch sequence). This can be achieved by changing the design of touch interactions by using more pressure gestures from pilots which might have as a result a reduction of the usability. Other engineering solutions might involve context information detection and fusing this information with operators' actions.

2) Interaction reliability and dependability (issue 8)

Finally, as for other avionic applications there is a need to apply verification and validation $(\mathrm{V} \& \mathrm{~V})$ techniques to the entire cockpit display system (from the input devices to the interactive multi-touch applications). This requires the engineering techniques used for the interactive multi-touch cockpits to be amenable to formal verification.

\section{RELATED WORK}

\section{A. Cockpits'interactivity}

Current (or soon to be released) interactive cockpits essentially feature both physical and WIMP interactions for triggering avionic functions. Indeed, when critical commands are considered, dependable interactions using physical interactors are preferred to software ones. Multi-touch applications on EFBs (Electronic Flight Bags) are already available as demonstrated by the Airbus application on the appStore [15]. Finally mono-touch interactions have already appeared in light aircrafts' cockpits and fighters [39] but multitouch applications for avionic application still remains at the level of industrial research projects such as [38].

\section{B. Notations for engineering multi-touch interactions}

Many research works have contributed to allow developers handling multi-modal interface design more easily including [27]. The domain specific language presented in [25], for instance, addresses some dimensions described in [19] as dynamic instantiation of input devices but lacks parallel behaviour description and interaction analysis. We observed that most notations (identified in [19]) lack the expressive power to encompass all the dimensions for multi-touch interactions and are not able to specify in a complete and nonambiguous way multi-touch (and more generally multi-modal) interactions. Proton++ [23] does not explain how to support multi-users touch interactions with several fingers. In conclusion, except the ICO notation (that is further described in next section), currently available notations for engineering multi-modal interactions, are not able to address all the engineering issues presented above.

\section{Development processes for interactive critical systems}

The DO-178C [12] defines the development process requirements for the development process of avionic software but does not detail how to address the specific issues of interactive software [28]. Only a few processes have targeted the development of interactive critical systems. Among such processes, [28] have defined the high level activities needed to take into account the system usability without sacrificing its reliability. Additionally, [18] proposed a refinement of the activities leading to the multi-touch interaction specification but lacks describing how to use the produced artefacts in the remainder of the development process.

\section{AN ARCHITECTURE}

This section proposes a layered architecture (presented Fig. 1) that ensures the availability of "good" properties of software systems mentioned in [25] (flexibility, separation of concerns, extensibility and hardware independence) and supports handling interactions with multiple fingers which corresponds to the dynamic instantiation of input devices (issue 2).

Fig. 1 describes an example of this architecture where the system is composed of two multi-touch sub-systems, one for each pilot. The physical interaction layers are designed to provide the system access to the users' events: fingers' touch on the display. These events are sent to hardware independent finger models which are registered to the interaction models. The fusion engine ensures the creation and forwarding of coherent interactions' events towards the applications (used by the pilots) as well as the communication between the two subsystems. To determine the coherence of the interactive events send to the applications, the fusion engine needs to know the characteristics of the widget on which the interaction takes place. 
This architecture is based on the work previously published in [19] which was an extension of another layered approach proposed in [13] but only dealing with multimodal interactions. We improved that architecture by introducing an intermediate level between the low-level transducer and the interaction models: a model to describe each finger behaviour. These dynamically instantiated models are independent from the lower layers and therefore ensure a higher flexibility and independence from the upper layers. In addition, we enriched the fusion engine with the capability to communicate with other systems. This communication capability via the Ivy bus [9] will be described more precisely in the case study section. The complete description of the other layers properties and communications have been introduced in [19] and are not repeated here due to space constraints. Finally, the JavaFx layer can be substituted to any other multi-touch programming language and the upper layers are notation independent.

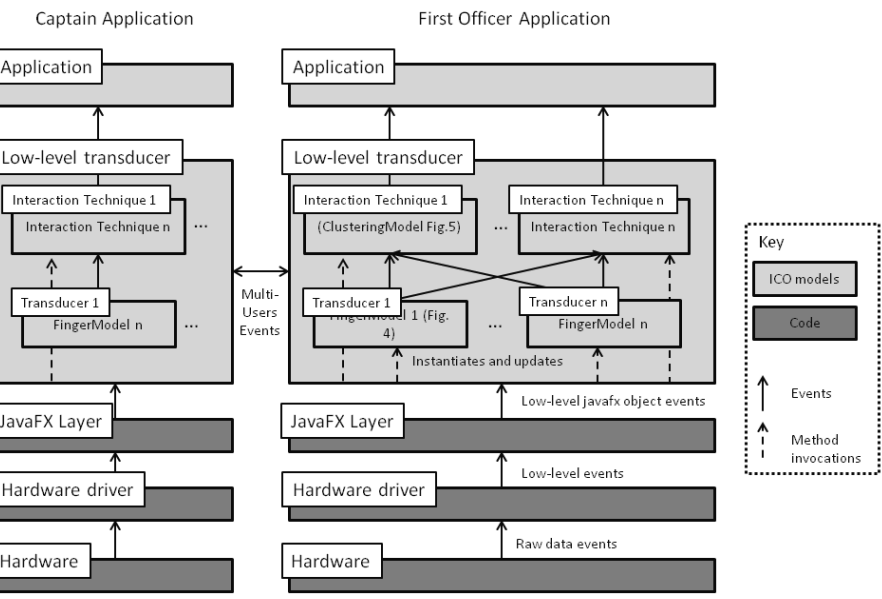

Fig. 1. Proposed layered architecture for multi-modal interaction

\section{A NOTATION FOR ENGINEERING MULTI-TOUCH INTERACTIONS}

\section{A. ICO: Informal definition}

The ICO notation (Interactive Cooperative Objects) is a formal description technique devoted to specify interactive systems. Using high-level Petri nets [16] for dynamic behaviour description, the notation also relies on objectoriented approach (dynamic instantiation, classification, encapsulation, inheritance and client/server relationships) to describe the structural or static aspects of systems.

ICO notation objects are composed of four components: a cooperative object for the behaviour description, a presentation part (i.e. Graphical Interface), and two functions (activation and rendering) describing the links between the cooperative object and the presentation part.

ICO addresses all the issues presented in the previous sections including dynamic instantiation of input devices which is not addressed elsewhere in a modelling technique. ICO uses the capabilities of Petri nets to describe tokens' creations which are used in models to represent input devices such as fingers on a touchscreen.

Lastly, it is important to note that ICOs have been used for various types of multi-modal interfaces [26] and in particular for multi-touch [19]. This notation is also currently applied to formally specify interactive systems in the fields of Air Traffic Management [30], satellite ground systems [33] and cockpits of military [6] and civil [5] aircrafts.

\section{B. Generalization of ICO event handling capabilities}

The models we present in this paper feature an extension of ICO that was required to address the issues of multi-touch interactions. In order to do so we proposed a new transition allowing one cooperative object to receive events. In models describing multi-touch interaction techniques, tokens may represent pilots' fingers "currently" on the device. These transitions can be used to specifically retrieve the input that emitted the event and to specify the event listened to as well as its source. It also exposes the properties of the event and allows specifying a condition on the event received in order to fire the transition. As this extension supersedes the previous event handler transitions in ICOs, the former transitions are no longer used.

Fig. 2 represents a use of this new mechanism: the model's extract presented is composed of a place OperationalInputDevices that contains the input devices of the system and their activation status. Such transition would belong to the Low-level transducer layer of Fig. 1. The disableInputDevice transition listens to "error" events emitted by the input devices. When the place OperationalInputDevices contains at least one active device, disableInputDevice is enabled. If so, when it receives an "error" event, disableInputDevice is fired if the criticality field of the "error" event is high (this is defined by the eventcondition level $==$ Level.Hight). When disableInputDevice is fired, the action (in Fig. 2 "device.idle()") is executed (setting the device in its idle mode) and the source device is moved to place NonOperationalInputDevices with the message received within the error event.

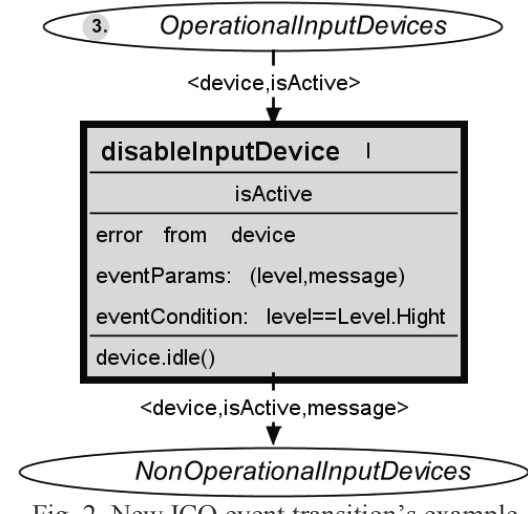

This ICO formalism extension enhances multi-modal interactions specification by offering the possibility to explicitly describe the events properties and their relationships with dynamically instantiated input devices.

\section{A tool to support the notation}

In order to edit and execute ICO models, the notation is supported by the CASE tool PetShop [18], [34]. The PetShop framework also provides tools to support user evaluations [32] and formal analysis of models [37] thus addressing issue 8 . 
In addition PetShop describes and simulates OS independent multi-touch interfaces (by connecting dedicated APIs to its kernel), which is not the case of most of the frameworks presented in [21].

\section{CASE STUDY}

The case study presented in this section focuses on demonstrating the expressive power of the ICO notation for the engineering of multi-modal and multi-touch applications. It also illustrate how the presented approach can be embedded in an industrial development process.

\section{A. Presentation}

This case study corresponds to a subset of a weather radar system from a civil aircraft cockpit which provides atmospheric data to the cockpit crew and is controlled by different systems:

- The input system is composed of a pushbutton on the glare shield panel: one on the Captain's (CPT) Flight Control Unit (FCU) and one on the First Officer's (FO) FCU and interactors on the pylon (duplicated for each pilot);

- The outputs of the weather radar are provided through the Navigation Displays (NDs);

- $\quad$ The back-up system, supported by the FCU-backup application is not part of the study. It provides pilots additional access to the weather radar controls based on this ARINC 661 User Application (UA).

The scenarios we present are representative of the potential use of multi-touch (modalities, users' interactions...) for such an application. However, we do not intend to address design aspects nor to demonstrate their operational validity as it is out of scope. Nevertheless, we intend to demonstrate that our notation provides a detailed and exhaustive mean to specify multi-touch critical systems and to cover all the issues presented above.

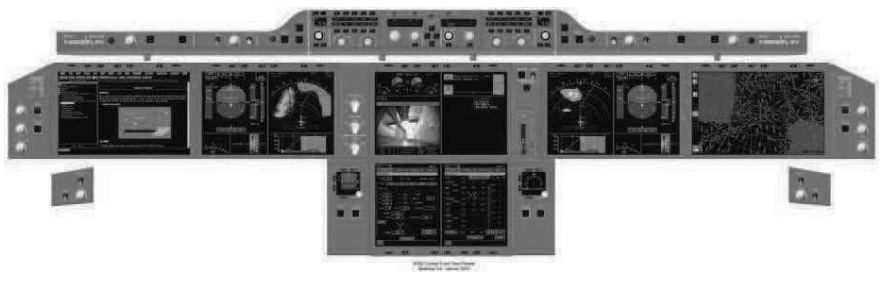

Fig. 3. A350 cockpit overview

For the purpose of this study, we consider a cockpit configuration in "T-shape" as of the Airbus A350 (Fig. 3). For the case study we consider a set of three displays: one screen in front of each pilot and one on the pylon, between them. These displays support multi-touch inputs (at least 5 touch inputs recognizable at the same time). The other technical features of the hardware are not considered here (resolution, latency...).

To illustrate the main dimensions of multi-modality, multitouch and multi-user interactions, we propose three pilot tasks belonging to different dimensions (see TABLE I. ).
TABLE I. PILOTS' TASKS AND THEIR DIMENSIONS

\begin{tabular}{|c|c|}
\hline Tasks & Dimensions \\
\hline $\begin{array}{c}\text { Basic weather-radar manipulations: } \\
\text { activate, change layer opacity }\end{array}$ & Single user, multi-touch \\
\hline Cloud evasive route, & Multi-users, synergistic \\
\hline $\begin{array}{c}\text { Modification of a "unique" weather } \\
\text { parameter by both pilots jointly }\end{array}$ & $\begin{array}{c}\text { Multi-touch, multi-user, } \\
\text { Exclusive }\end{array}$ \\
\hline
\end{tabular}

To support these three tasks we have set up a platform composed of three PCs running PetShop and linked using the Ivy Bus [9], one ND application on each pilot's computer and a validation application for both of them to interact on. Such architecture is compliant with the work done in [14] where each PetShop is running over an ARINC 653 operating system to address dependability issues (not addressed here).

Fig. 4 presents the multi-touch interface displayed on each pilot's ND. The interface in composed of a multi-touch map and various custom widgets emulating physical buttons and interactors that command the display modes and the weather radar controls.

\section{1) Task 1: Advanced multi-touch manipulation}

For this task, a pilot (either the CPT or the FO) interacts with the weather radar through the multi-touch Navigation Display to (de)activate its visualization or modify its intensity (opacity of the layer displaying the weather information on top of the ND's map). In addition, the pilot is able to interact with the map itself: modifying the range (zoom) level and translate the map.

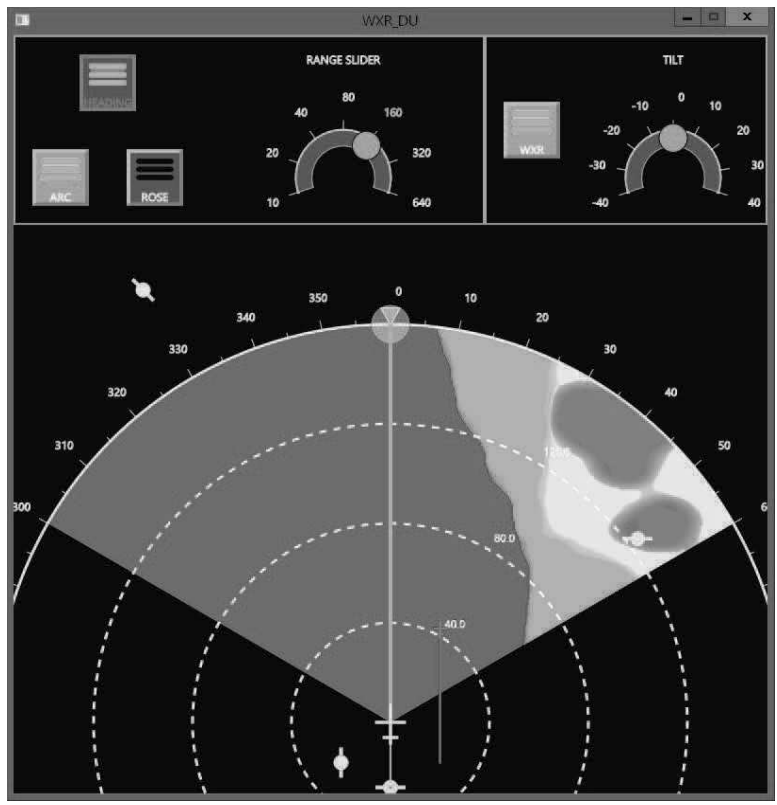

Fig. 4. Navigation Display's interface for this case study

These interactions have been designed and proposed in various research papers as well as public SDKs (Software Development kits) of multi-touch platforms. TABLE II. summarizes theses interactions and their references. They cover issues 1, 2, 3 and 4 described above and are covered by models belonging to the logical interaction layer of Fig. 1. 
TABLE II. A SAMPLE OF MULTI-TOUCH INTERACTIONS FOR WEATHER RADAR MANIPULATION

\begin{tabular}{|c|c|c|c|}
\hline $\begin{array}{c}\text { Interaction } \\
\text { technique }\end{array}$ & $\begin{array}{l}\text { Command } \\
\text { description }\end{array}$ & $\begin{array}{c}\text { Interaction } \\
\text { properties targeted }\end{array}$ & Reference \\
\hline $\begin{array}{l}1 \text { finger } \\
\text { drag }\end{array}$ & $\begin{array}{l}\text { Change } \\
\text { Heading }\end{array}$ & Direct Manipulation & Most SDKs \\
\hline $\begin{array}{l}1 \text { finger } \\
\text { lasso }\end{array}$ & $\begin{array}{c}\text { Select object } \\
\text { on map }\end{array}$ & Selection & {$[25]$} \\
\hline $\begin{array}{l}2 \text { fingers' } \\
\text { flick }\end{array}$ & $\begin{array}{c}\text { Trigger } \\
\text { weather layer } \\
\text { visibility }\end{array}$ & $\begin{array}{l}\text { Two fingers with same } \\
\text { unitary interaction, } \\
\text { velocity and } \\
\text { acceleration }\end{array}$ & Most SDKs \\
\hline $\begin{array}{l}\text { Precise } \\
\text { Tilt/Z- } \\
\text { transition }\end{array}$ & $\begin{array}{c}\text { Tune weather } \\
\text { layer } \\
\text { intensity }\end{array}$ & $\begin{array}{l}\text { Timing aspects, multi- } \\
\text { fingers }(>2)\end{array}$ & {$[24],[17]$} \\
\hline $\begin{array}{l}2 \text { fingers' } \\
\text { rotate }\end{array}$ & Map rotation & $\begin{array}{l}\text { Direct Manipulation } \\
\text { (continuous) }\end{array}$ & Most SDKs \\
\hline $\begin{array}{l}2 \text { fingers' } \\
\text { scale }\end{array}$ & Map range & $\begin{array}{l}\text { Direct Manipulation } \\
\text { (continuous) }\end{array}$ & Most SDKs \\
\hline
\end{tabular}

We modelled each interaction technique in TABLE II. using the ICO notation and implemented them in our application according to the architecture presented Fig. 2. In this paragraph, we emphasize ICO's expressive power by describing an excerpt of the precise tilt interaction technique (also referred as "Z-Translation" in [24]), and more precisely the behaviour part regarding fingers movements on the touchscreen. [24] describes this interaction technique as follows: " $z$ translation - a conjoined touch on the object, together with a one-touch drag up and down".

This designer-like description remains at a very high level and when developers have wish to implement such interaction techniques, several cases need further details as, for instance, the description of the impact of each finger movement on the screen.

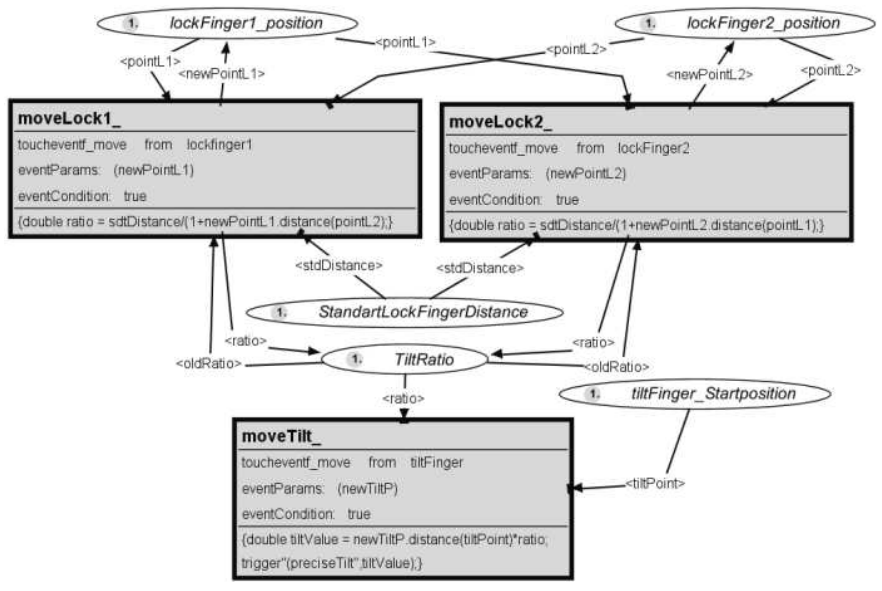

Fig. 5. Excerpt of the model of the behaviour of "tilt"

Fig. 5 describes a simple case where the third finger (controlling the tilt value) is moving. When is does so, the transition moveTilt is fired and executes its code, triggering a "preciseTilt" event. The tilt value only takes into account the distance between the initial touch of the tilt finger and its current position. As a refinement, designers may need to modify this behaviour by weighting this value with the distance between the two fingers serving as anchors. The model presented in Fig. 5 illustrates how events' handling mechanisms in ICO can describe that feature with a rather simple and localized modification of the original model.

\section{2) Task 2: Cooperative cloud-avoiding manoeuvers}

This paragraph details a synergistic task between both pilots consisting in avoiding clouds during flight. While the captain is responsible for flying the aircraft from a short term perspective using the weather radar at close range, the first officer is studying the weather further away along the predetermined flight path to avoid potentially uncomfortable zones for the passengers. The first officer then proposes a new route via his ND. Both pilots must then validate the flight plan modification on the confirmation display, each one having to press his validation button at the same time.

Fig. 6 describes the behaviour of the validation application which is symmetrical for both pilots. When the FO proposed a new flight plan, the newFlightPlanFromFO transition is fired and a token containing a reference towards the FO's ND and the proposed flight plan is put in place NeedOfValidation. When the FO (resp. CPT) presses his validation button, the transition FOValidate (resp. CPTValidate) is fired and a new token is put in FO interacts (resp. CPT interacts). The CPT (resp. FO) has to validate within $2.5 \mathrm{~s}$ otherwise the validation is not allowed. If the FO (resp. CPT) released the button before the CPT (resp. FO) validates, he has to start over the validation. If the proposal is not validated within ten seconds, it is discarded, discardValidationRequest is fired and the FO's ND is notified accordingly.

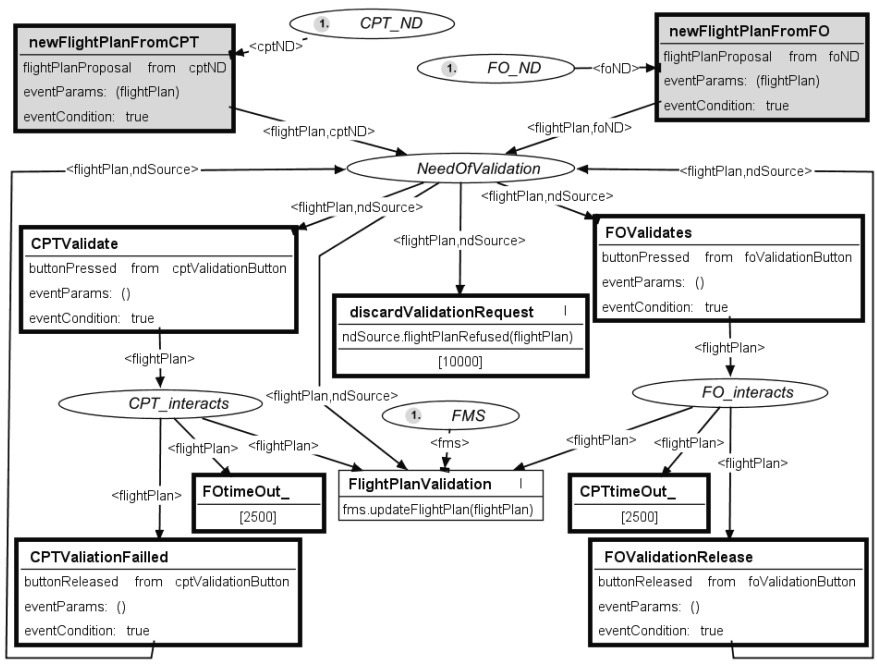

Fig. 6. Flight Plan cooperative validation model

3) Task 3: Concurrent edition of a singleton parameter

This third task illustrates the interaction conflicts that may occur as both pilots modify simultaneously the same system parameter. For this task, both pilots try to modify the tilt angle of the weather radar simultaneously.

Such conflicts are likely to appear as more and more system parameters are controlled by User Applications which are easily replicated on multiple displays. In the latest Airbus cockpits, when a pilot is moving his KCCU's cursor over a system page, conflicts are avoided by denying the other pilot's cursor access to that DU area. This approach is fundamentally opposed to the one proposed in [22] where conflicts are likely 
to be solved by human rather than locking mechanisms. Therefore they propose an optimistic algorithm for conflict resolution favouring interactions as much as possible and providing undo mechanisms when a seldom conflict occurs.

Our approach implements the main principle supported by [22]: "User commands must be handled immediately without waiting for either authorizations or acknowledgments".

Let us consider the interactor commanding the tilt angle of the weather radar on the captain side. Because denying both pilots interacting over the same system page is very restrictive, one might want to only prevent them from interacting on the same widget. Fig. 7 presents an excerpt of the behavioural model of such an interface. This model is identical for both pilots. Since all DUs and their UAs are connected, the place Peer contains a reference of the FO's ND applications. When a pilot starts interacting on the widget controlling the weather radar's tilt angle, this widget w (of which a reference is stored in place TiltWidget) triggers an "interactionStarting" event. If the tilt is not locked for this pilot, the event is received via the canReserve transition. This transition locks the tilt on the other pilot's application using the service lockTilt() preventing the other pilot to interact with his own tilt widget. At the end of the interaction, the model updates the tilt on the other application and allows the other pilot to interact with it.

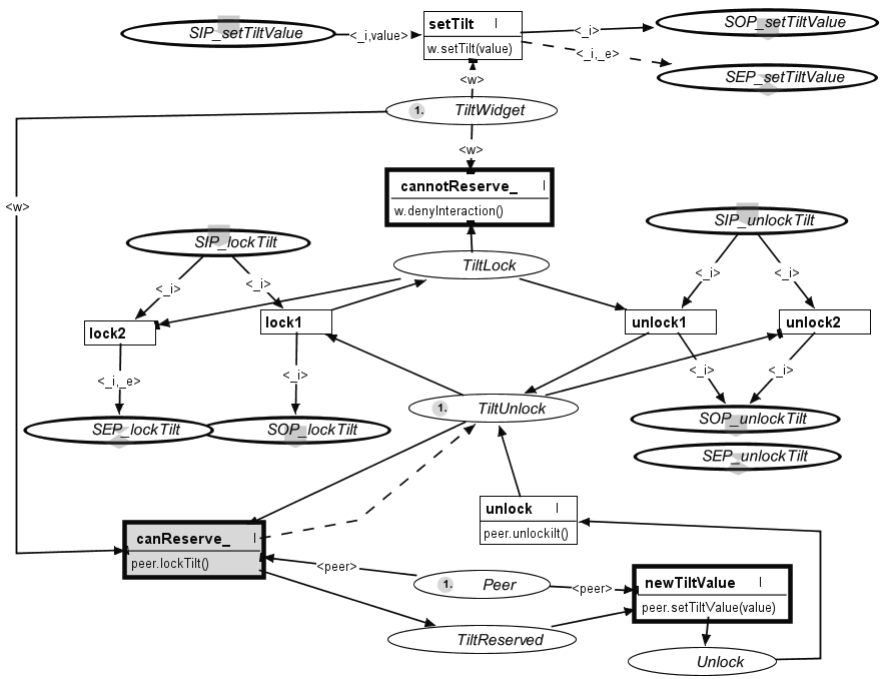

Fig. 7. Excerpt of the ND application model

The dashed arcs represent exception arcs formally described in [7]. Such an exception can be thrown by the FO's ND application if the FO is interacting with the tilt widget. The Captain model represents how this exception can be caught making it possible to specify what behaviour follows its occurrence: this could be denying captain's interactivity or notifying the conflict to him.

\section{B. A notation embedded in an development process}

In this section, we illustrate how the proposed notation can be used within an industrial development process for embedded critical systems. The development process described in Fig. 8 is derived from the standard V development process currently applied in the aeronautic and compliant with the DO$178 \mathrm{C}$ [12] requirements and with the process presented in [28].
The particularity of this process lies in the use of the artefacts produced during the interaction design and modelling phase. The formal specification (ICO models) that have been created for prototyping and operational concepts validation can be reused all along the path down to the suppliers. The suppliers can then base their implementation on the ICO models, serving as specification artefacts. These models will then be used during the validation phase of the produced software which ensure the implementation behave as specified. By reducing the numbers of artefacts in the development process, we reduce the developing costs and possible loss of specification items during the development.

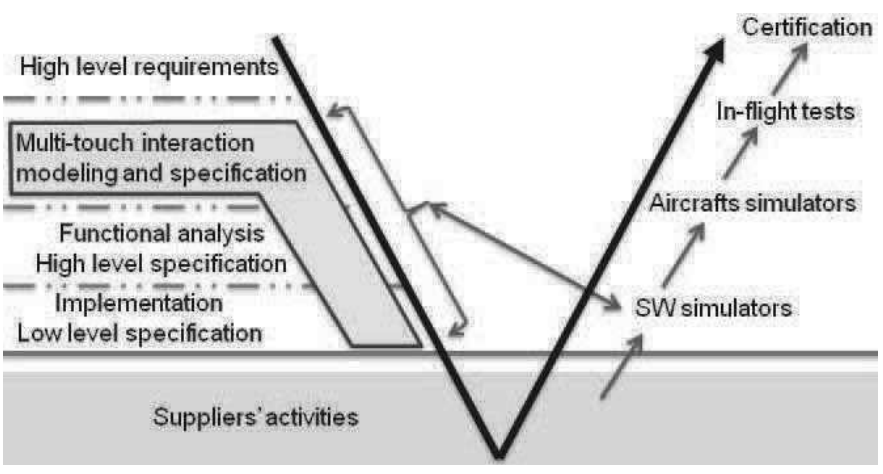

Fig. 8. Example of aeronautical development process

\section{CONCLUSION}

This paper has presented the extensions and the use of a formal description technique [19] for the detailed specification of multi-touch and multi-user interfaces. It has built upon previous work done in the area of low-level interaction modelling as proposed in [1] and [2]. It also extended the layered architecture proposed in [26] to address the specific issues of touch interactions. The paper has provided a combination of an architecture and a notation which also enables addressing the outputs of an interactive critical system in a similar way as what was presented with input. Through a set of representative tasks, we have demonstrated on a case study how the ICO notation can tackle multi-modal and multiusers issues providing a detailed and legible notation for interactive critical system specification. All the models presented in the paper run in PetShop environment and (provided the paper is accepted) the presentation will demonstrate their execution on a multi-touch platform. Finally we illustrated how the notation can enhance an industrial development process for interactive critical systems.

This work is part of a more ambitious work trying to identify the modifications that would have to be added to actual A661 specification standard to address multi-touch and multi-users interactions.

\section{REFERENCES}

[1] Accot J., Chatty S., Palanque P. A formal description of low level interaction and its application to multimodal interactive systems. DSVIS 96, Springer Verlag, (1996), 156-175.

[2] Accot, J., Chatty, S., Maury, S., Palanque, P.: Formal Transducers: Models of Devices and Building Bricks for Highly Interactive Systems. DSVIS 97, Springer, Heidelberg (1997), 23-37. 
[3] ARINC 661-5, Prepared by Airlines Electronic Engineering Committee. Cockpit Display System Interfaces to User Systems. ARINC Specification 661-5; (2013)

[4] Barbé J., Wolff M. \& Mollard R.. 2013. Human centered design approach to integrate touch screen in future aircraft cockpits. In Proc. 15th int. conf. (HCI Int. '13), Springer-Verlag, 429-438

[5] Barboni E., Conversy S., Navarre D. \& Palanque P. Model-Based Engineering of Widgets, User Applications and Servers Compliant with ARINC 661 Specification. 13th conf. on Design Specification and Verification of Interactive Systems (DSVIS 2006), LNCS Springer Verlag. p25-38

[6] Bastide R., Navarre D., Palanque P., Schyn A. \& Dragicevic P. A Model-Based Approach for Real-Time Embedded Multimodal Systems in Military Aircrafts. Sixth International Conference on Multimodal Interfaces (ICMI'04) October 14-15, 2004, USA, ACM Press

[7] Bastide R., Sy O. \& Palanque P. A formal notation and tool for the engineering of CORBA systems. Concurrency - Practice and Experience 12(14): 1379-1403 (2000)

[8] Bojan Blazica, D. V. \& Mladenic V.D. HDCMD: A Clustering Algorithm to Support Hand Detection on Multitouch Displays. SouthCHI 2013: 803-814

[9] Buisson M, Bustico A, Chatty S., Colin F-R., Jestin Y., Maury S., Mertz C. \& Truillet P. Ivy: un bus logiciel au service du développement de prototypes de systèmes interactifs. Proc. of Conférence Francophone sur l'Interaction Homme-Machine (IHM '02). 2002 ACM DL, 223-226.

[10] Coutaz, J., Nigay, L., Salber, D., Blandford, A., May, J., \& Young, R. 1995. Four Easy Pieces for Assessing the Usability of Multimodal in Interaction: the CARE Properties. Human Computer Interaction, Interact' 95. Chapman \& Hall (IFIP); 95:pp. 115-120.

[11] Dang C-T., Straub M., \& André E. Hand distinction for multi-touch tabletop interaction. Proc. of the ACM International Conference on Interactive Tabletops and Surfaces (ITS '09) 2009.

[12] DO-178C Software Considerations in Airborne Systems and Equipment Certification: Radio Technical Commission for Aeronautics (RTCA) European Organization for, 2011

[13] Echtler F. \& Klinker G.. 2008. A multitouch software architecture. In Proc. of the 5th Nordic Conf. on Hum.-Comput. Interact: building bridges (NordiCHI '08). ACM, 463-466

[14] Fayollas C., Fabre J-C., Navarre D., Palanque P. \& Deleris Y. FaultTolerant Interactive Cockpits for Critical Applications: Overall Approach. Software Engineering for Resilient Systems (SERENE 2012), Springer-Verlag, p. 32-46, 2012

[15] FlySmart with Airbus EFB Manager - Retrieved: 2013-11-24. Available at: $\quad$ https://itunes.apple.com/us/app/flysmart-airbus-efbmanager/id605062108? $\mathrm{mt}=8$

[16] Genrich, H. J. 1991. Predicate/Transitions Nets. In High-Levels Petri Nets: Theory and Application. K. Jensen and G. Rozenberg, Springer Verlag (1991) pp. 3-43

[17] GestureWorks - true Multitouch for Flash. Website. Retrieved: 2013 11-08. Available at: http://gestureworks.com/

[18] Hamon A., Palanque P., Deleris Y., Navarre D. \& Barboni E.. A Toolsupported Development Process for Bringing Touch Interactions into Interactive Cockpits for Controlling Embedded Critical Systems. Int Conf. on Hum.-Comput. Interact. in Aeronautics (HCI'Aero 2012), ACM DL, p. 25-36, 2012

[19] Hamon A., Palanque P., Silva J-L., Deleris Y., \& Barboni E. Formal description of multi-touch interactions. 2013 Proc. 5th ACM symposium on Engineering interactive computing systems (EICS '13). ACM, $207-$ 216

[20] Hutchins E. \& Lauwsen T. Distributed Cognition in an airline cockpit. In Cognition and Communication at work (Engeström \& Middleton Eds.), Cambridge University Press, 1996

[21] Kammer D., Keck M., Freitag G. \& Wacker M. Taxonomy and Overview of Multi-touch Frameworks: Architecture, Scope and Features. In Proc. of Workshop on Engineering Patterns for Multi-Touch Interfaces, Berlin, Germany, June 2010.
[22] Karsenty A., Beaudouin-Lafon M. An Algorithm for Distributed Groupware Applications. ICDCS 1993: 195-202

[23] Kenrick K., Björn Hartmann, Tony DeRose, and Maneesh Agrawala. 2012. Proton++: a customizable declarative multitouch framework. In Proceedings of the 25th annual ACM symposium on User interface software and technology (UIST '12). ACM, 477-486.

[24] Kenrick K., Miller T., Bollensdorff B., DeRose T., Hartmann B. \& Agrawala M.. Eden: a professional multitouch tool for constructing virtual organic environments. 2011 Proc. of conf. on Human factors in computing sys. (CHI '11). ACM, 1343-1352.

[25] Khandkar S.H. \& Maurer F. A domain specific language to define gestures for multi-touch applications. In Proceedings of the 10th Workshop on Domain-Specific Modeling (DSM '10). ACM, 6 pages.

[26] Ladry J-F., Navarre D., Palanque P. Formal description techniques to support the design, construction and evaluation of fusion engines for sure (safe, usable, reliable and evolvable) multimodal interfaces. ICMI 2009: $185-192$

[27] Lü H. \& Li Y. Gesture studio: authoring multi-touch interactions through demonstration and declaration. 2013 Proc. of SIGCHI Conf. on Human Factors in Computing Sys. (CHI '13)

[28] Martinie,C. Palanque P., Navarre D., and Barboni E.. 2012. A development process for usable large scale interactive critical systems: application to satellite ground segments. Proc. 4th int. conf. on HumanCentered Software Engineering (HCSE'12) Springer-Verlag, 72-93.

[29] Micire M., Desai M, Drury J-L., McCann E., Norton A., Tsui K. M. \& Yanco H.A. Design and validation of two-handed multi-touch tabletop controllers for robot teleoperation. Proc. of the 16th international conference on Intelligent user interfaces (IUI '11). ACM, 145-154.

[30] Navarre D., Palanque P., Ladry J-F. \& Barboni E. ICOs: A model-based user interface description technique dedicated to interactive systems addressing usability, reliability and scalability. ACM Trans. Comput.Hum. Interaction, 16(4), 18:1-18:56. 2009

[31] NBAA 2013: Touchscreen Cockpit Systems and Next Generation Business Aircraft - Online Article from Aviation Today - Retrieved: 20153-02-21. Available at: http://www.aviationtoday.com/av/topstories/NBAA-2013-TouchscreenCockpit-Systems-and-Next-Generation-Business-Aircraft_80495.html

[32] Palanque P., Barboni E., Martinie C., Navarre D., Winckler M: A model-based approach for supporting engineering usability evaluation of interaction techniques. EICS 2011: 21-30

[33] Palanque P., Bernhaupt R., Navarre D., Ould M. \& Winckler M. Supporting Usability Evaluation of Multimodal Man-Machine Interfaces for Space Ground Segment Applications Using Petri net Based Formal Specification. Ninth Int. Conference on Space Operations, 2006

[34] Palanque P., Ladry J-F, Navarre D. \& Barboni E. High-Fidelity Prototyping of Interactive Systems can be Formal too 13th Int. Conf. on Hum.-Comput. Interact. Springer Verlag, LNCS 5610

[35] Quinn P., Malacria S. \& Cockburn A. Touch scrolling transfer functions. 2013 Proc. of ACM symposium on User interface software and technology (UIST '13). ACM, 61-70.

[36] Shneiderman B. (1983). Direct manipulation: a step beyond programming languages, IEEE Computer 16(8), 1983, 57-69.

[37] Silva J-L., Fayollas C., Palanque P. \& Barboni E. Analysis of WIMP and Post WIMP Interactive Systems based on Formal Specification, Proceedings of the 5th International Workshop on Formal Methods for Interactive Systems (FMIS 2013)

[38] The Cockpit of 2030 - Retrieved: 2013-11-24. Available at http://onboard.thalesgroup.com/2010/intuitive-innovative-interactivethe-cockpit-of-2030/

[39] Touch Screens Are Tested for Piloting Passenger Jets - Retrieved: 201311-24. Available at:

http://www.nytimes.com/2013/07/06/technology/passenger-jets-testingtouch-screen-technology.html? r=0

[40] van Dam A. 1997. Post-WIMP user interfaces. Commun. ACM 40, 2 (February 1997), 63-67 